\title{
Obesity and respiratory function
}

\section{Chiovato}

Monaldi Arch Chest Dis 2006; 65: 2, 67-68.

Correspondence: Luca Chiovato, MD, Ph.D.; Professor of Endocrinology, University of Pavia; Head Unit of Internal Medicine and Endocrinology; Salvatore Maugeri Foundation, Institute of Pavia; e-mail: lchiovato@fsm.it

Two papers published in the current issue of Monaldi Archives address the problem of obesity, both from a diagnostic and therapeutic point of view. Obesity has become epidemic in developed countries, with the increasing prevalence of this morbid condition expected to reach nearly $50 \%$ by the year 2025 in North America and Europe. The epidemic of obesity is not the result of a viral contagion or a of "new" endocrine disease involving metabolism, but rather a manifestation of the evolutionary principle of "survival of the fittest", that is now misplaced in time. Obesity and its genes are no longer advantages for a world that has more than sufficient food for most, and little need to expend energy to obtain it. Also, the human lifespan is no longer only a few decades, instead it often reaches eight or nine decades. This means that the comorbid effects of excess adipose tissue accumulate, often becoming life-threatening. As the severity of conditions of overweight and obesity increase, so do the incidences of diabetes, hypertension, coronary artery disease, strokes, congestive heart failure, hepatocellular and biliary diseases, endocrine abnormalities, psychosocial disorders, orthopedic problems, as well as the risk of certain malignancies. Contributing to some of these relationships are the prevalent lipid abnormalities and the obstructive sleep apnea that are seen in obese patients.

Orthopnea, effort dyspnea and expiratory flow limitation (EFL) are frequently observed in obese patients even without bronchial obstruction. The availability of a simple tool for evaluating EFL in obesity would allow a better understanding of the dyspnea in these patients. The negative expiratory pressure (NEP) test, a commonly used method for investigating EFL, might lead to misleading results in obese patients, due to excessive collapsing of the upper airways. Partial submaximal flow/volume curves (PSCs) might be more appropriate for evaluating EFL in obese patients, because this test is not influenced by the upper airway collapsibility. In their study Drigo et al. [1] evaluated PSCs as a tool for understanding EFL in obese patients, and investigated the relationship between the shape of the flow/pressure diagram of quiet breathing airway resistance (Raw-qb) and EFL detected by PSCs. Obese patients (BMI > $30 \mathrm{~kg} / \mathrm{m} 2)$ without bronchial obstruction, but with suspected EFL underwent PSCs and the Raw-qb test. The main result was that in nearly $30 \%$ of investigated obese patients, PSCs did not confirm the spirometric suspicion of EFL, a strong indication for EFL being restricted to $25 \%$ of them, who would need confirmation by a gold standard method. Moreover, a loop in the Raw-qb expiratory diagram was significantly more frequent in subject diagnosed by PSCs as having EFL. These, together with previous findings from the same group of investigators, suggest that the expiratory reserve volume (ERV) could play an important role in the genesis of EFL in obese patients, and that short elderly females with obesity could be at greater risk for EFL and, more importantly, for clinical problems related to EFL. While the paper of Drigo et al. [1] would help understanding the mechanisms responsible for dyspnea in obese patients, two major limitations should be considered: failure to compare results with a gold standard for EFL and inclusion of obese patients who received spirometric test for respiratory complains. Thus, future investigations are needed to ascertain whether the present findings might be extended to the universe of obese patients.

Few medical problems are as frustrating to treat as obesity. With the remarkable exception of bariatric surgery, which is often hampered by major side effects, approaches to lifestyle (e.g. hypocaloric diets, behavior, physical activity) and currently available drug therapy are poorly effective, mainly due to extremely high rates of recidivism for reduced-obese patients. Therapeutic results are even more discouraging in view of the notion that a weight reduction of only $10 \%$, if maintained over the time, may be enough to have a substantial benefit on the patient's health. In this issue of the Monaldi Archives, the therapeutic results of an interdisciplinary rehabilitation programme in patients with morbid obesity (BMI $>35 \mathrm{~kg} / \mathrm{m}^{2}$ ) were evaluated in a 6-month prospective observational study by Clini et al. [2]. The interdisciplinary conservative treatment was delivered to patients hospitalised for 1 month, and included: i) a polysomnographic assessment of apnea/hypopnea (AHI) followed by appropriate nCPAP correction, if needed; ii) low-calorie diet and nutritional education; iii) incremental exercise sessions; and iv) psychotherapy and behavioral therapy. The main results were: i) resting $\mathrm{PaO}_{2}$ significantly rose over the time and AHI decreased, these favourable outcomes being maintained for up to 6 months; ii) body weight (BW) significantly decreased during 
the hospitalisation period. At the 6-month followup evaluation, $41 \%$ of patients showed a further $>5 \% \mathrm{BW}$ decrease, $24 \%$ a $<5 \% \mathrm{BW}$ decrease, $5 \%$ remained stable, and $24 \%$ regained BW; iii) exercise capacity, as assessed by a 6-minute walking distance test, significantly improved over all of the follow-up; iv) overall quality-of-life scores significantly improved at 1 month, but reverted to baseline at 6 months with the exception of sleep efficiency, problem solving, and social interaction subscales; v) the percentage of patients with high Binge Eating Scores decreased from $88 \%$ to $51 \%$ after 6 months; vi) glycosylated hemoglobin and total cholesterol did improve, but their changes were more impressive at 1 month than at 6 months. These encouraging results, obtained in patients with extreme degrees of obesity, should be compared with those reported by Golay et al. [3] using an interdisciplinary cognitive-behavioral-nutritional therapy, combined with daily physical activity and relaxation sessions on weight and psychosocial issues, during a 6-week in-hospital stay. At the time of discharge from hospital, patients had lost an average $7.6 \mathrm{~kg}$. At the end of a 5-year follow-up, $25.5 \%$ of them had lost a further 11.9 $\mathrm{kg}$ (mean), 20\% maintained their initial weight loss, but $54.5 \%$ regained weight $(10.4 \mathrm{~kg})$. Thus, compared to the Swiss trial, the study by Clini et al. [2] has the main limitation of a much shorter follow-up period. Although both studies were nonrandomised and included a limited number of patients, they provide relevant indications for the effectiveness of interdisciplinary rehabilitation programs in morbid obese patients.

While the results of these treatment approaches are promising, a major drawback might derive from their economical burden. For example, in
Italy the costs of a long (4- to 6-week) in-hospital stay would not be covered by the National Health Service reimbursement for "acute" patients with severe obesity, which currently scores as "high" as 1.400-2.100 Euro in different Italian Regions. It follows that these rehabilitation programs would require special reimbursement rates. This might be possible if Regional and Central Government Agencies recognised the epidemic of obesity with its dramatic impact on morbidity and mortality as a major health problem. During the first half of the last century, special health regulations were enacted in Italy in order to limit and treat the epidemic of tuberculosis. These regulations involved establishing dedicated hospitals or hospital departments, as well as outpatients' clinics for the diagnosis and treatment of tuberculosis. Thus, the question might be raised whether, in the first half of the present century, the time is ripe to ask for special health regulations regarding obesity and its treatment.

\section{References}

1. Drigo R, Coin A, Fontana M, Enzo E, Ferraresso A, Zambotto F. Partial flow/volume curves and airway resistances in obese subjects: new tools for investigating flow limitation. Monaldi Archives 2006; 65: 117-121.

2. Clini E, Prato F, Nobile M, Bondi M, Serri B, Cilione C, Lugli D. Interdisciplinary rehabilitation in morbidly obese subjects: an observational pilot study. Monaldi Archives 2006; 65: 89-95.

3. Golay A, Buclin S, Ybarra J, Toti F, pichard C, Picco $\mathrm{N}$, de Tonnac N, Allaz AF. New interdisciplinary cognitive-behavioral-nutritional approach to obesity treatment: a 5-year follow-up study. Eat and Weight Disorders 2004; 9: 29-34.

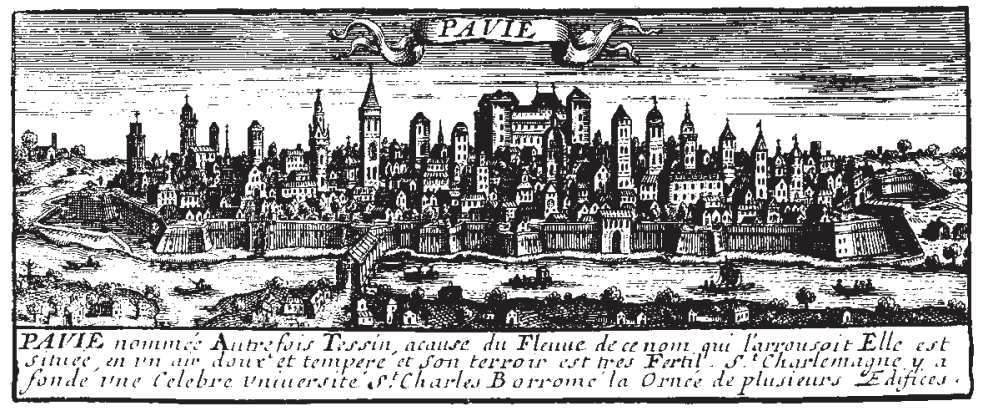

